\title{
Video Article \\ Differential Imaging of Biological Structures with Doubly-resonant Coherent Anti-stokes Raman Scattering (CARS)
}

\author{
Tyler J. Weeks ${ }^{1}$, Thomas R. Huser ${ }^{1,2}$ \\ ${ }^{1}$ Center for Biophotonics, University of California, Davis \\ ${ }^{2}$ Department of Internal Medicine, University of California, Davis
}

Correspondence to: Thomas R. Huser at trhuser@ucdavis.edu

URL: https://www.jove.com/video/2085

DOI: doi:10.3791/2085

Keywords: Cellular Biology, Issue 44, Raman scattering, Four-wave mixing, Coherent anti-Stokes Raman scattering, Microscopy, Coherent Raman Scattering

Date Published: 10/17/2010

Citation: Weeks, T.J., Huser, T.R. Differential Imaging of Biological Structures with Doubly-resonant Coherent Anti-stokes Raman Scattering (CARS). J. Vis. Exp. (44), e2085, doi:10.3791/2085 (2010).

\section{Abstract}

Coherent Raman imaging techniques have seen a dramatic increase in activity over the past decade due to their promise to enable labelfree optical imaging with high molecular specificity ${ }^{1}$. The sensitivity of these techniques, however, is many orders of magnitude weaker than fluorescence, requiring milli-molar molecular concentrations ${ }^{1,2}$. Here, we describe a technique that can enable the detection of weak or low concentrations of Raman-active molecules by amplifying their signal with that obtained from strong or abundant Raman scatterers. The interaction of short pulsed lasers in a biological sample generates a variety of coherent Raman scattering signals, each of which carry unique chemical information about the sample. Typically, only one of these signals, e.g. Coherent Anti-stokes Raman scattering (CARS), is used to generate an image while the others are discarded. However, when these other signals, including 3-color CARS and four-wave mixing (FWM), are collected and compared to the CARS signal, otherwise difficult to detect information can be extracted ${ }^{3}$. For example, doubly-resonant CARS (DR-CARS) is the result of the constructive interference between two resonant signals ${ }^{4}$. We demonstrate how tuning of the three lasers required to produce DR-CARS signals to the $2845 \mathrm{~cm}^{-1} \mathrm{CH}$ stretch vibration in lipids and the $2120 \mathrm{~cm}^{-1} \mathrm{CD}$ stretching vibration of a deuterated molecule (e.g. deuterated sugars, fatty acids, etc.) can be utilized to probe both Raman resonances simultaneously. Under these conditions, in addition to CARS signals from each resonance, a combined DR-CARS signal probing both is also generated. We demonstrate how detecting the difference between the DR-CARS signal and the amplifying signal from an abundant molecule's vibration can be used to enhance the sensitivity for the weaker signal. We further demonstrate that this approach even extends to applications where both signals are generated from different molecules, such that e.g. using the strong Raman signal of a solvent can enhance the weak Raman signal of a dilute solute.

\section{Video Link}

The video component of this article can be found at https://www.jove.com/video/2085/

\section{Protocol}

\section{Generation of CARS and DR-CARS Signals}

In order to generate CARS and DR-CARS signals simultaneously, three tunable and synchronized short pulsed laser sources are required.

1. To obtain three synchronized pulses we start with a single $10 \mathrm{~W}$ laser (PicoTrain, HighQ laser, Inc.). This laser has a fixed wavelength of 1064 $\mathrm{nm}$, a fixed pulse length of $7 \mathrm{ps}$, and a fixed repetition rate of $76 \mathrm{MHz}$.

2. Using a series of half-wave plates and polarizing beam splitter cubes the beam is split into three parts. A half-wave plate combined with a polarizing beam splitter cube allows us to adjust the amount of power in each component of the beam without changing its direction. Typically, two beams are adjusted to $\sim 4.5 \mathrm{~W}$ each, and the third beam contains the remaining $1 \mathrm{~W}$.

3. The two high power beams are then directed into two independent optical parametric oscillators (OPO's, Avante, APE GmbH, Berlin, Germany). OPO's use difference frequency generation to convert a higher energy photon into two lower energy photons with different wavelengths. By controlling the temperature of the crystal used to achieve this effect, the wavelengths of the resulting photons can be controlled to within $0.1 \mathrm{~nm}$. By frequency-doubling these signals, a laser with a fixed wavelength of $1064 \mathrm{~nm}$ can now be transformed into a laser beam that can be tuned anywhere between $780 \mathrm{~nm}$ to $910 \mathrm{~nm}$. By pumping two separate OPO's with the same $1064 \mathrm{~nm}$ source, we obtain two independently tunable laser sources that are automatically synchronized to our original pump laser.

4. The third (lowest power) beam from the $1064 \mathrm{~nm}$ pump laser is directed around the OPO's by a combination of dichroic mirrors so that all three beams ( 2 from the OPOs, 1 from the pump laser) can be recombined later. In order to efficiently produce coherent Raman signal photons the recombined pulses must be overlapped both temporally and spatially. Because OPO's contain ring cavities, allowing each laser pulse to pass through the crystal multiple times, the beams sent through the OPO's travel an extra distance resulting in a considerable delay relative to the original pump beam. This distance must be compensated for with the third beam by introducing extra mirrors that force this beam to travel the same distance as the other two before they are recombined using dichroic mirrors. 
5. In order to provide fine adjustment of the length of the path each beam travels adjustable delay stages, using prism retroreflectors, are introduced into each beam path.

6. Another set of half-wave plates and polarizing beam splitter cubes are added to each beam to allow us to adjust the power of each beam independently without affecting the input of the OPO's.

7. Dichroic mirrors are used to first combine the beams from the two OPO's and then with the $1064 \mathrm{~nm}$ beam. Care must be taken to ensure that the beams are precisely overlapped (i.e. they are collinear and have similar divergence). This can be checked by comparing the overlap of the beams close to (within a few centimeters) and far from $(\sim 1 \mathrm{~m})$ the dichroic mirror.

8. The advantage of using pulsed lasers is that each pulse can have a high peak energy at the sample, allowing for efficient generation of coherent Raman signals, while still maintaining a low average intensity. A high average intensity can damage the sample. Therefore in order to further control the average intensity without sacrificing peak energy, the three combined beams are sent through an electro-optic modulator (Pockel's cell, ConOptics) functioning as a pulse picker, which allows us to adjust the repetition rate of the pulses that arrive at our sample and therefore the average intensity.

9. The combined laser beams are then coupled into an inverted microscope with a high NA objective. The objective is typically a $60 \mathrm{X}$ water objective lens with a numerical aperture (NA) of 1.2 that has been corrected for chromatic aberration across the range of tunability of our OPO's and the $1064 \mathrm{~nm}$ beam. The tight focusing generated by the high NA objective lens allows for the most efficient generation of coherent Raman signals on the micron scale.

10. The combined beams are expanded in order to overfill the back-port of the microscope objective. By overfilling the back of the microscope objective we can achieve the best focusing conditions and therefore the best spatial resolution in our microscope system.

11. The objective lens in our microscope is mounted on an XYZ piezo stage which allows us to obtain images by raster-scanning the beams across the sample, similar to commercial beam-scanning confocal microscopes.

\section{The Use of Three Short Pulsed Lasers}

The use of three short pulsed lasers results in the production of several CARS signals, from the various combinations of two lasers, as well as 3Color CARS and DR-FWM signals from the combination of all three lasers.

1. The wavelengths of the signals can lie spectrally close to each other. When the signals are close together it can be difficult to separate them for analysis using fixed wavelength bandpass filters and dichroic mirrors. For this reason our signals are passed into an imaging spectrometer (SpectraPro 2300i, Acton Research) that also serves as an efficient monochromator to spatially separate signals at different wavelengths.

2. An electronically actuated flip mirror within the spectrometer in the lowered position sends the signal to a back-illuminated deep-depletion charge-coupled device (CCD) camera which provides spectroscopic information across the entire signal range and allows us to identify and optimize the various coherent Raman signals.

3. To select the signal with which we wish to image we simply rotate the grating within the spectrometer, using the vendor-supplied control and data acquisition software (WinSpec, Princeton Instruments), to center the peak of interest on the CCD camera and then change the position of the flip mirror to redirect this signal to a second exit port to which a single-photon counting avalanche photo-diode (APD) has been attached.

4. The objective lens is then raster-scanned and the signals recorded on the APD are used to generate an image by displaying the photon count-rate for each pixel via data acquisition software (SymPhoTime, Picoquant GmbH, Berlin, Germany).

5. We repeat this imaging procedure for each desired coherent Raman signal which allows us to compare the signals during post-processing.

\section{Sample Preparation}

In order to obtain clear, reproducible images some care must be taken in preparing the sample.

1. Samples are typically prepared on $\sim 150$ micron thick glass coverslips. These coverslips are thin enough to allow for high resolution imaging with the 1.2 NA objectives that are typically used.

2. Optical trapping of dielectric objects can occur when the laser light is diffracted through small transparent objects. The use of tightly focused, high peak power, short pulsed laser beams can then drag small cells or bacteria along, resulting in blurred or smeared images. In order to avoid this it may be necessary to immobilize the sample on the surface of the glass coverslip by first applying a thin layer of poly-L-lysine by spin-coating.

3. For cells in culture glass bottom culture dishes can be utilized that allow for imaging without the need to detach the cells from their cell culture growth dishes.

4. For demonstration in this contribution we first deposit a formaldehyde-fixed C. elegans nematode worm on a glass cover slip.

5. Then add a $20 \mu \mathrm{L}$ droplet of $5 \mathrm{M}$ deuterated glucose solution to the worm. The deuterated glucose solution provides a unique and strong Raman background signature.

\section{Sample Analysis}

In order to properly take advantage of the doubly-resonant enhancement effect the Raman spectra of both Raman-resonant substances must be known.

1. Once the sample is prepared on the coverslip it is analyzed using a confocal Raman microscope to obtain the relevant spontaneous Raman spectrum and identify suitable peaks.

2. To perform DR-CARS we identify the spectral locations of the $\mathrm{CH}$ stretch mode, typically associated with lipids, and the $\mathrm{CD}$ stretch mode, associated with the deuterated glucose, to be $2845 \mathrm{~cm}^{-1}$ and $2121 \mathrm{~cm}^{-1}$ respectively.

3. Coherent Raman scattering is achieved when the frequency difference between two lasers matches the frequency of a molecular vibration. By tuning one OPO to $817 \mathrm{~nm}$ it will probe the $2845 \mathrm{~cm}^{-1} \mathrm{CH}$ mode when combined with the $1064 \mathrm{~nm}$ laser beam and by tuning the other OPO to $868 \mathrm{~nm}$ it will probe the $2121 \mathrm{~cm}^{-1}$ peak when combined with the $1064 \mathrm{~nm}$ beam. 
4. By raster-scanning the sample on the CARS microscope we can now observe three coherent Raman signals of interest. A CARS signal probing the $\mathrm{CH}$ stretching vibration, a CARS signal probing the CD stretch mode, and a DR-CARS signal probing both.

5. We select each peak as described in Section 2.3 and take an image as described above.

\section{Image Processing}

Extracting additional information based on these three images now requires some fairly simple image processing.

1. First the images must be normalized. In theory normalization can be achieved by accounting for the intensity of each laser involved in each signal generation process. In practice, however this does not always work, mainly due to the non-uniform spectral response of dichroic mirrors, detectors, and gratings.

2. A practical method for normalization relies on the fact that in regions where pure lipids dominate the signal, the $C D$ resonance should not contribute to any of the signal. Likewise, in regions of pure glucose solution the $\mathrm{CH}$ resonance should not contribute to any of the signal. With this in mind, we normalize the DR-CARS image and the CARS image obtained on the CD resonance of the deuterated glucose solution to a region well outside the $C$. elegans worm that should exhibit no $\mathrm{CH}$ resonance.

3. Then we identify a region within the worm in the $\mathrm{CH}$-resonant CARS image that is rich in lipids and normalize it to the corresponding region within the normalized DR-CARS image. For this method to work properly we assume that deep within this region no deuterated glucose is present. This is a safe assumption since the lipids are hydrophobic and will not mix with the solution.

4. Now, by subtracting the normalized $\mathrm{CH}$-resonant CARS image from the normalized DR-CARS image we are left with just the amplified CDresonant signal.

5. Similarly by subtracting the normalized CD-resonant CARS image from the normalized DR-FWM image we are left with just the amplified CHresonant signal.

\section{Representative Results}

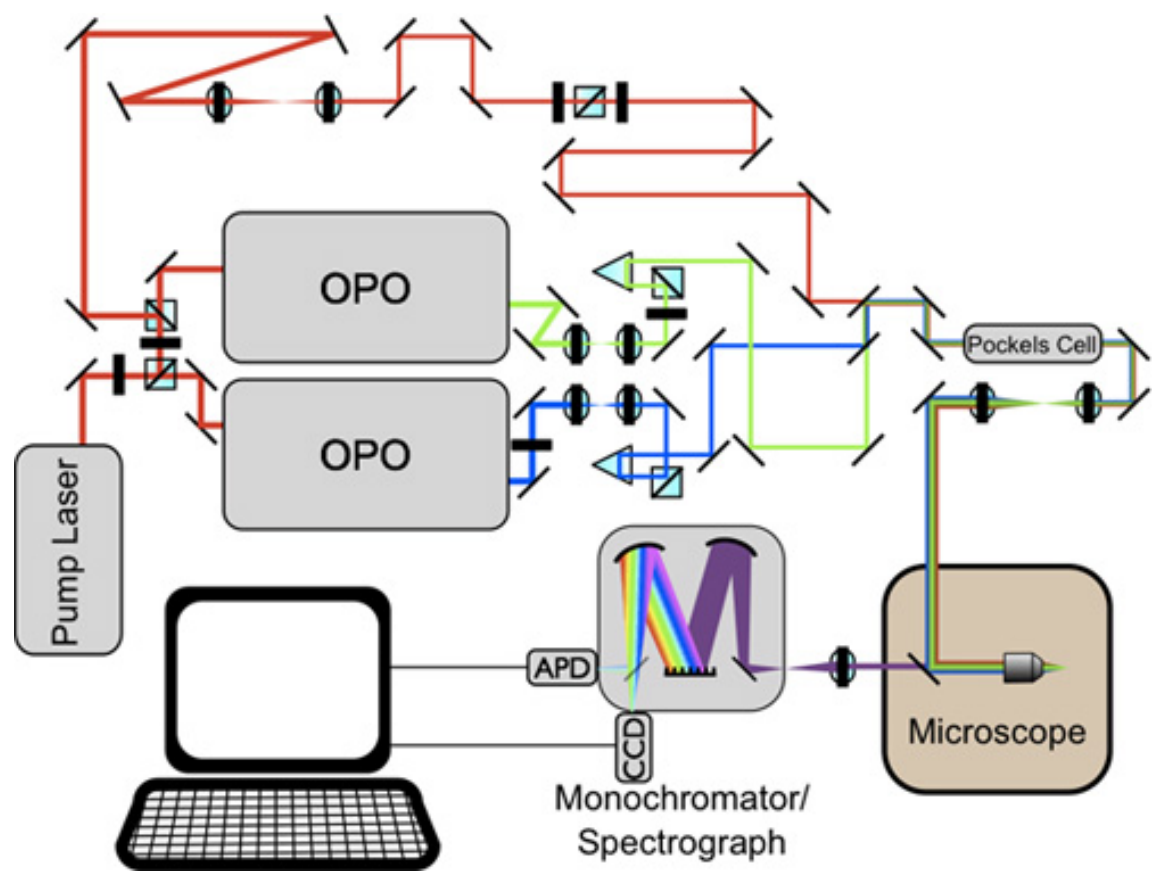

Figure 1: Diagram of DR-CARS microscopy system as described above. 


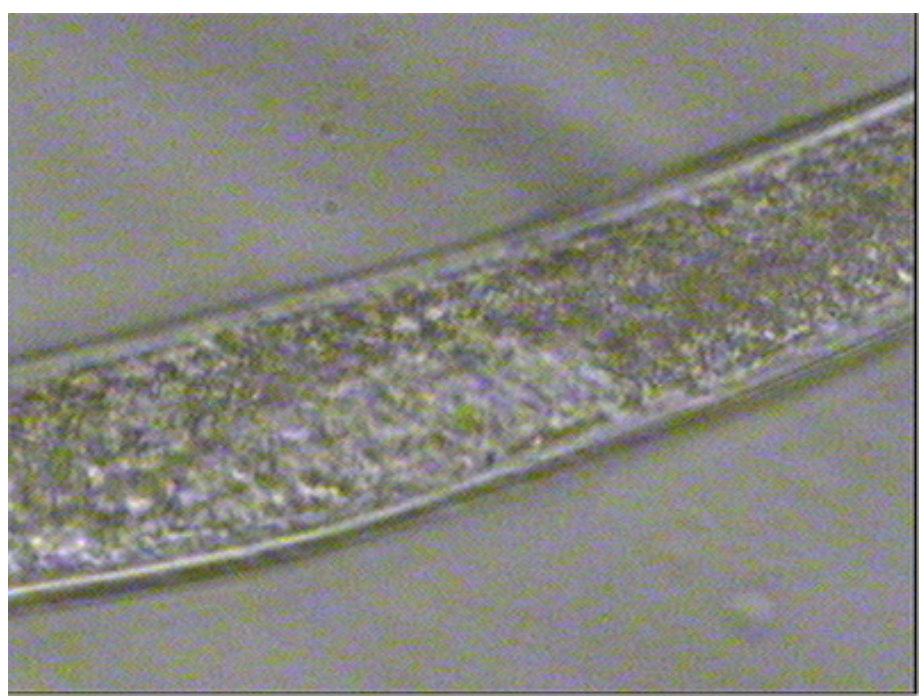

Figure 2: White light image of a C. elegans worm in deuterated glucose solution prepared on a glass coverslip and ready for imaging.

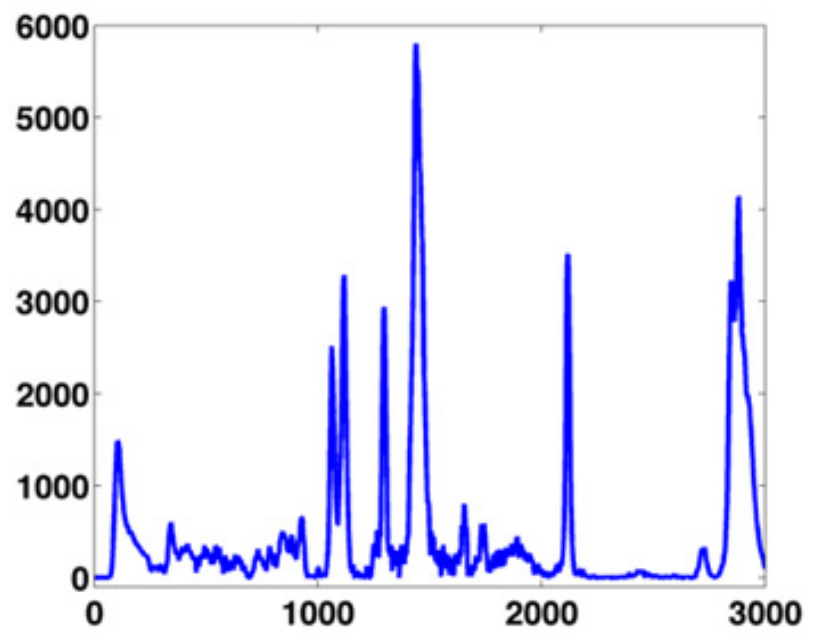

Figure 3: Raman spectrum of modified oleic acid (an unsaturated fatty acid) that includes an alkyne modification (a carbon triple-bond carbon group). The strong $\mathrm{CH}$ resonances at $2845 \mathrm{~cm}^{-1}$ and the alkyne resonance at $2100 \mathrm{~cm}^{-1}$ are both well isolated from the fingerprint region (the region of densely packed peaks), making them ideal markers for coherent Raman imaging. 


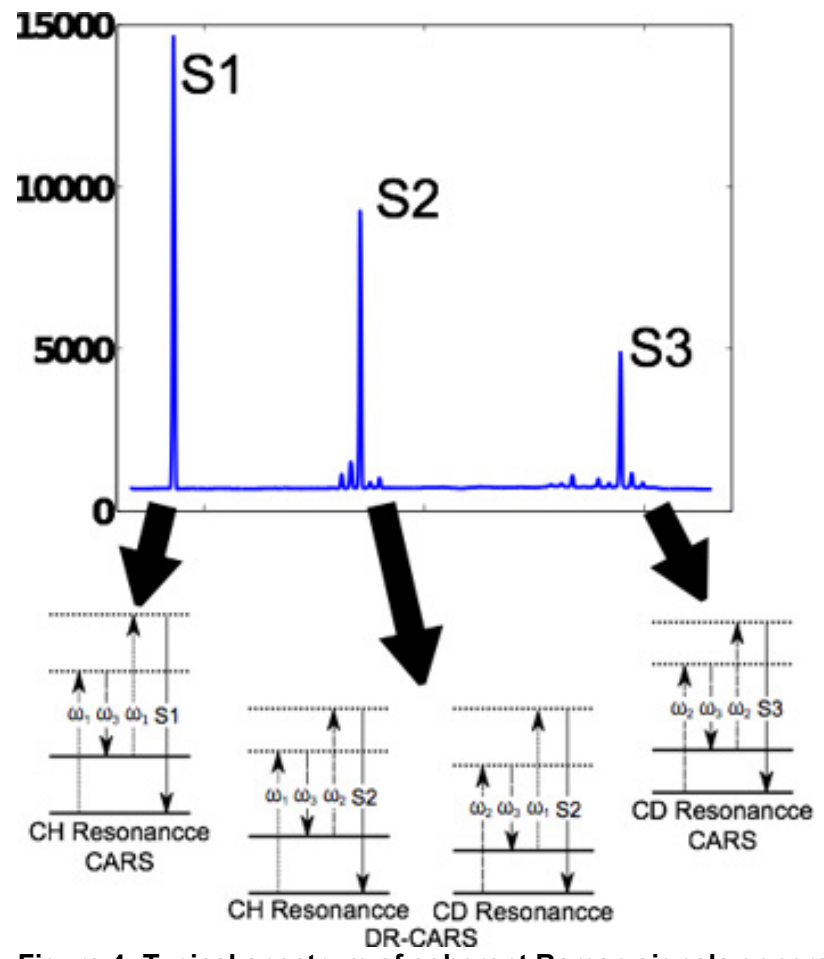

Figure 4: Typical spectrum of coherent Raman signals generated when three short-pulsed lasers are overlapped within the sample. The arrows point to the process(es) responsible for each signal as represented by energy diagrams. In the diagrams shown here dashed arrows indicate photons from the laser and the OPO's and solid arrows indicate the resulting signal. The solid horizontal lines indicate the energy of the Raman vibration and give a visual representation that, in DR-CARS, mixing the same 3 input photons simultaneously probes two different Raman vibrations.

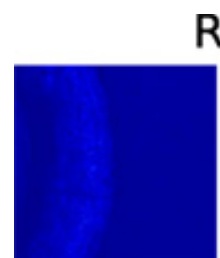

CH Resonance CARS

\section{Raw Images}

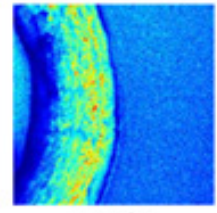

Both DR-CARS

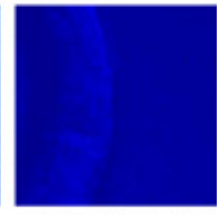

CD Resonance CARS

\section{Normalized Images}

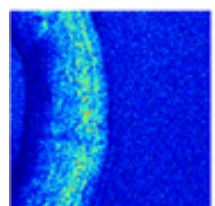

CH Resonance CARS

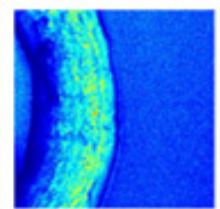

Both

DR-CARS

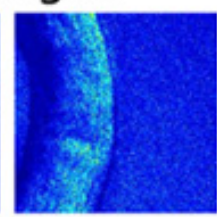

CD Resonance CARS

\section{Difference Images}

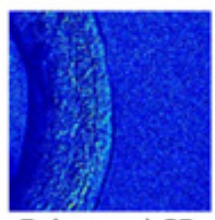

Enhanced CD

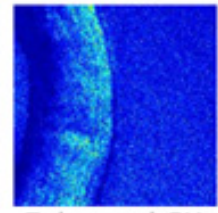

Enhanced $\mathrm{CH}$

Figure 5: Typical results from imaging $\boldsymbol{C}$. elegans worms using DR-CARS and CARS. The top row used the three signals indicated in Figure 4 to image a worm in a solution of deuterated glucose. In the second row the images were appropriately normalized and in the third row the difference images were produced by subtracting each of the CARS images from the DR-CARS image. 


\section{Discussion}

Raman spectroscopy and Raman-based imaging are powerful emerging tools in the bio sciences. Currently, this is particularly true for the in vivo and in vitro study of cellular metabolism and metabolic disorders in processing and storage of lipids. Most bio-macromolecules contain a large number of similar, mostly carbon-based molecular bonds, so that the Raman spectra obtained from cells and organisms are typically a convolution of contributions from lipids, proteins, nucleic acids, sugars, etc. Lipids are relatively easy to isolate from these complex spectra, because of their tendency to form dense droplets or bilayers and because they contain extended chains with a large number of aliphatic $\mathrm{CH}$ bonds. Our ability to isolate specific proteins, amino acids, RNA, or DNA within the complex cellular environment is, however, very limited. This is particularly true if these molecules of interest are only present at $\mu \mathrm{M}$ concentrations and below. Here, the ability to probe weak Raman resonances utilizing our newly introduced DR-CARS difference imaging technique provides a potentially powerful approach for their chemical microanalysis and imaging. Admittedly, the most complicated portion of this protocol is the alignment and synchronization of the laser system. When starting from scratch, the synchronization of the pulses, i.e. ensuring that the pulses are overlapped in time despite the different paths they take can be facilitated by the use of a pulse autocorrelator. Once spatial and temporal overlap is achieved, CARS and DR-CARS signals should be readily detectable. However, the first alignment is often crude, resulting in weak signals. The best practice for aligning this system well is to initially generate weak signals and then to improve the signal strength by gently tweaking the mirrors along each path and adjusting the temporal overlap using the delay stages. Although the spectrometer/monochromator acts as a very efficient baffle for room light the cleanest results can be achieved by operating the system with the room lights turned off and curtains or lens tubing to minimize background introduced by the various other light sources (e.g computer monitors, indicator lights, LEDs, etc.).

Our particular setup utilizes single-photon counting avalanche photo-diode (APD) detectors and time-correlated single photon counting (TCSPC) hardware for detection ${ }^{5}$. This enables us to detect extremely weak signals with relatively low noise but many groups have found photo-multiplier tubes (PMT's) with variable gain advantageous when making similar measurements. The advantage of PMT's is that they offer variable gain and have a much larger detection area which can simplify alignment of the detector. Additionally, our setup utilizes piezo stages to translate the objective in order to achieve beam scanning. The advantage of this is that we have the ability to return to any spot within the previously scanned image with a high degree of accuracy and take additional measurements including spontaneous Raman spectra. Other groups have been successful utilizing scanning mirror assemblies, or even entire confocal scanning units such as the Olympus FluoView system, which offers much faster imaging but is limited in its ability to precisely return to arbitrary locations within an image.

Tuning the lasers to match the Raman resonance is also a critical step that may require some optimization. Although the Raman peaks may be known the maximum spectral peak intensity obtained from DR-CARS and CARS does not necessarily correspond to the maximum of the spontaneous Raman peak. This is due to the intrinsic interference of signals generated by four-wave mixing leading to a non-resonant background signal and CARS, which distorts CARS spectra relative to spontaneous Raman spectra. The spectral location of the peak of the CARS signal can be calculated, but a more practical approach is to tune the OPOs in several, small spectral steps across the expected location of the Raman resonance. This process should yield a clear maximum. In fact, for the greatest sensitivity from DR-FWM both resonances must be tuned to this maximum.

One last potential problem of the DR-CARS approach has to also be discussed, i.e. the DR-CARS signal will depend on a homogeneous distribution of the Raman-active amplifying molecule. For most biological objects, this could well be the broad OH resonance from water, which is abundant and almost omnipresent. Water is, however, excluded from hydrophobic regions with a cell, such as lipid droplets, leading a distortion of the signals obtained when utilizing the water resonance to amplify lipid modes. In our example, we have used a solution of deuterated glucose to generate an easily detectable and abundant signal for our biological sample. Similarly, deuterated water or deuterated biological buffers, such as d-HEPES could be used. In our example, the lipid droplets within the $C$. elegans worm were small enough to always contain both, the deuterated glucose solution and lipids within the focused laser spot of our system. This, however, is not generally true. A particular example would be adipocytes, which generate rather large lipid droplets within their cytoplasm. This means, any experiment conducted with the DR-CARS technique requires careful preparation and control experiments to verify the results.

\section{Disclosures}

No conflicts of interest declared.

\section{Acknowledgements}

We would like to thank Iwan Schie and Sebastian Wachsmann-Hogiu for their contributions in developing the DR-CARS technique. Tyler Weeks acknowledges support by the Lawrence Scholar Program from Lawrence Livermore National Laboratory. Thomas Huser is grateful for support from the American Heart Association through the Grant-in-Aid program. This work was also supported in part by funding from the National Science Foundation. The Center for Biophotonics, an NSF Science and Technology Center, is managed by the University of California, Davis, under Cooperative Agreement No. PHY 0120999. Support is also acknowledged from the UCD Clinical Translational Science Center under grant number UL1 RR024146 from the National Center for Research Resources (NCRR).

\section{References}

1. Cheng, J. X., Volkmer, A., \& Xie, X. S. Theoretical and experimental characterization of coherent anti-Stokes Raman scattering microscopy. J Opt Soc Am B 19 (9) (2002).

2. Tolles W. M., Nibler, J. W., \& McDonald, J. R., Harvey, A. B. A Review of the Theory and Application of Coherent Anti-Stokes Raman Spectroscopy (CARS). Appl Spectrosc (1977). 
3. Weeks, T., Schie, I. W., Wachsmann-Hogiu, S., \& Huser, T. Signal generation and Raman-resonant imaging by non-degenerate four-wave mixing under tight focusing conditions. J Biophoton 3 (3)169-175 (2010).

4. Weeks, T., Wachsmann-Hogiu, S., \& Huser, T. Raman Microscopy based on Doubly-Resonant Four-Wave Mixing (DR-FWM). Opt Express 17 (19) 17044-17051 (2009).

5. Schie, I. W., Weeks, T., McNerney, G. P., Fore, S., Sampson, J. K., Wachsmann-Hogiu, S., Rutledge, J. C., Huser, T. Simultaneous forward and epi-CARS microscopy with a single detector by time-correlated single photon counting. Optics Express 16 (3) $2168-2175$ (2008). 